\title{
B cell depletion: a novel therapy for autoimmune diabetes?
}

\author{
Hélène Bour-Jordan and Jeffrey A. Bluestone
}

Diabetes Center, Department of Medicine, and Department of Pathology, UCSF, San Francisco, California, USA.

\begin{abstract}
Autoimmune diabetes is believed to be mediated primarily by $T$ cells. However, $B$ cells have been implicated in the pathogenesis of the disease in NOD mice. Although preclinical studies have been limited by the absence of anti$C D 20$ reagents that can induce $B$ cell depletion in mice, a clinical trial using the $\mathrm{B}$ cell-depleting anti-CD20 monoclonal antibody rituximab (Rituxan) is underway in type 1 diabetes patients. In this issue of the JCI, Hu et al. describe the generation of transgenic NOD mice that express human CD20 on $B$ cells (see the related article beginning on page 3857 ). They show that anti-CD20 therapy induces $B$ cell depletion in these mice and offers some level of protection against diabetes. Although many questions remain unanswered, this mouse model represents the first opportunity to evaluate the potential value of rituximab as a novel therapy for autoimmune diabetes.
\end{abstract}

Type 1 diabetes (T1D) is an autoimmune disease targeting insulin-producing $\beta$ cells in the pancreatic islets of Langerhans. Despite years of fundamental and clinical research, no cure has been found for this devastating disease. Thus, there is a growing interest in immunotherapies approved for other diseases that could be rationally investigated in T1D patients. In this regard, rituximab (Rituxan; Genentech), a humanized anti-CD20 mAb that induces $B$ cell depletion and is FDA approved for B cell lymphoma therapy, is currently being assessed in a clinical trial in T1D. However, until recently, preclinical studies have been limited by the absence of antiCD20 reagents that induce $B$ cell depletion in mice. In this issue of the JCI, Hu et al. address this issue by generating transgenic NOD mice that express human CD20 on B cells (hCD20/NOD mice) (1). Treatment with a murine anti-CD20 $\mathrm{mAb}$ that targets the same epitope as rituximab rapidly induced complete B cell depletion in lymph nodes and bone marrow and partial depletion in the spleen, albeit with limited effects in the peritoneal cavity. Importantly, anti-CD20 therapy partially protected prediabetic hCD20/NOD mice from disease and restored euglycemia in one-third of

Nonstandard abbreviations used: ADCC, antibodymediated cellular cytotoxicity; hCD20, human CD20; T1D, type 1 diabetes.

Conflict of interest: The authors have declared that no conflict of interest exists.

Citation for this article: J. Clin. Invest. 117:3642-3645 (2007). doi:10.1172/JCI34236. new-onset diabetic mice. Furthermore, Hu et al. suggest that protection from disease is associated with the generation of Treg and regulatory B cell populations. Thus, the study by Hu et al. provides a proof of principle that supports the investigation of anti-CD20-targeting therapies in T1D, and the transgenic mouse created by these authors provides a useful model in which to examine further the mode of action of rituximab and second-generation antiCD20 drugs in vivo.

\section{Anti-CD20 therapy in autoimmunity}

$\mathrm{CD} 20$ is a $35-\mathrm{kDa}$ transmembrane protein expressed on immature $B$ cells beginning at the pre-B cell stage, on all mature $B$ cells with the exception of plasma cells, and on B cell lymphomas. Rituximab is a chimeric murine/human $\mathrm{mAb}$ targeting CD20 that is FDA approved for the treatment of non-Hodgkin B cell lymphomas and induces rapid B lymphoma depletion. There has been a growing interest in the use of rituximab for autoimmune diseases, since it induces depletion of normal $\mathrm{B}$ cells and is generally a well-tolerated drug (reviewed in ref. 2). Rituximab was first evaluated in human autoimmune diseases in which B cells and autoantibodies play a direct pathogenic role. Rituximab has shown some efficiency in experimental trials of chronic idiopathic thrombocytopenia, antineutrophil cytoplasmic autoantibody-positive Wegener granulomatosis, and autoimmune hemolytic anemia and in phase I/II open-label trials in systemic lupus erythematosus (3-7). Importantly, randomized controlled trials have shown clear benefits of rituximab in rheumatoid arthritis and in ongoing multiple sclerosis trials, both autoimmune diseases in which $\mathrm{T}$ and $\mathrm{B}$ cells are involved, similar to T1D $(8,9)$. Although initial results have been encouraging in clinical settings in which rituximab has been used to date, many basic questions remain unanswered, such as the optimal dose and regimen, whether rituximab should be used in combination with other therapies to obtain the most favorable outcome, the mode of action of rituximab, and its effect on the immune system beyond B cell depletion.

\section{Role of $B$ cells}

\section{in autoimmune diabetes}

The NOD mouse is a well-characterized model of autoimmune diabetes that is believed to exhibit many of the mechanisms underlying the pathogenesis of the human disease (10), and as such, it is likely instructive in examining the role of B cells in T1D. Mononuclear infiltrates in both NOD mouse and human islets comprise mainly $\mathrm{CD} 4^{+}$and $\mathrm{CD} 8^{+} \mathrm{T}$ cells, but $\mathrm{B}$ cells, NK cells, dendritic cells, and macrophages have been described (10-12). The central role of $\mathrm{T}$ cells in autoimmune diabetes was demonstrated in the NOD model by the ability of $\mathrm{T}$ cells to transfer disease and the protection afforded by immunotherapies targeting $\mathrm{T}$ cells (13-16). The precise function of autoreactive B cells has been more difficult to establish (Figure 1). Isletspecific autoantibodies targeting major autoantigens such as glutamic acid decarboxylase and insulin can be detected in the serum in NOD mice and in patients and have been an excellent indicator of autoimmunity and predictor of disease in diabetic patients and relatives $(17,18)$. However, a direct pathogenic role for autoantibodies is controversial. Greeley et al. reported that maternal autoantibodies are important for the development of disease in NOD mice (19), but diabetes cannot be transferred by autoantibodies. In NOD mice, the impor- 


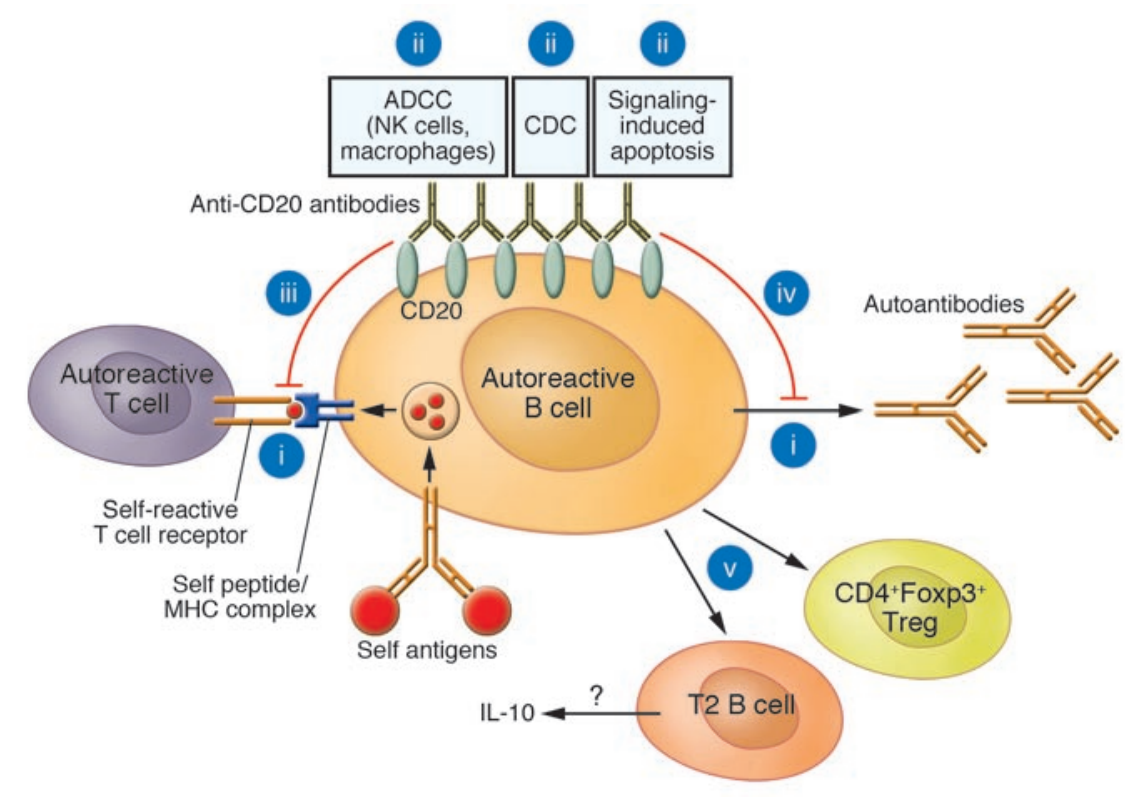

Figure 1

Anti-CD20 therapy and B cells in autoimmunity. Autoreactive B cells play a role in autoimmune diseases via their production of circulating autoantibodies and/or their role as antigen-presenting cells for autoreactive $T$ cells after the capture of self antigens by cell surface autoantibodies that increase their antigen-presentation capabilities (i). Rituximab and other anti-CD20 mAbs crosslink CD20 on the surface of $B$ cells and induce B cell depletion mainly through ADCC, although complement-dependent cytotoxicity (CDC) and apoptosis have also been implicated (ii). AntiCD20-mediated B cell depletion prevents interaction with autoreactive T cells (iii) and reduces the amount of circulating autoantibodies (iv), although with much slower kinetics. Finally, as suggested by Hu et al. in their study in this issue of the $\mathrm{JCl}(1)$, anti-CD20 therapy may induce Treg and regulatory $\mathrm{B}$ cell populations (CD4+Foxp3 ${ }^{+}$Tregs and transition type 2 [T2] B cells) that could play a role in restoring immune tolerance, possibly via the production of IL-10 (v).

tance of B cells was clearly demonstrated by the dramatic reduction in insulitis and diabetes incidence following B cell depletion using anti-IgM antibodies at birth or in NOD mice genetically deficient in B cells (20). Evidence from many different studies suggests that B cells function as islet antigen-presenting cells for autoreactive T cells in NOD mice and that autoantibodies expressed on the cell surface improve the capture and presentation of autoantigens (21-24). Importantly, early loss of B cells does not eliminate insulitis in NOD mice, suggesting that autoreactive $\mathrm{B}$ cells may be essential at late stages of disease to enhance autoreactivity and promote epitope spreading $(20,21)$. This finding has significant clinical implications, since B cell-depleting therapies such as rituximab would be most useful in patients newly diagnosed with T1D or islet transplant recipients (25).

Novel mAbs targeting murine CD20 and inducing $\mathrm{B}$ cell depletion have been developed recently (26), and transgenic mice expressing hCD20 in B cells have been generated on the FVB mouse genetic back- ground (27) and now by Hu et al. (1) on the NOD background. Importantly, the extent and tissue variability of $\mathrm{B}$ cell depletion following anti-CD20 therapy appears similar in these models, suggesting that they may provide clinically relevant information. Most significantly, $\mathrm{Hu}$ et al. showed that anti-CD20 therapy in new-onset diabetic hCD20/NOD mice reversed diabetes in one-third of the treated mice, providing a much-needed experimental rationale for the investigation of rituximab in $\mathrm{T} 1 \mathrm{D}$, especially since clinical trials are already underway (28). It is unclear why only a third of mice became euglycemic, but the protocol followed by Hu et al., which consisted of waiting six days after diagnosis of diabetes before starting treatment, a time point when most residual islets have been destroyed, could have contributed to the low success rate. Indeed, in another therapeutic setting, we showed that it is critical to target mice with blood glucose lower than $350 \mathrm{mg} / \mathrm{dl}$ (29). Interestingly, in the $\mathrm{Hu}$ et al. study, the reversal of hyperglycemia was characterized by variable and gen- erally slow kinetics but long-term effect. This could reflect the presence of two separate processes: a short-term antiinflammatory effect in islets, whose kinetics might depend on the aggressiveness of the autoimmune infiltrate, and a slower induction of regulatory cell population(s) that could induce long-term tolerance.

\section{Mechanism of action of anti-CD20 therapy in NOD mice}

Little is known about the mechanisms underlying the efficacy of "normal" B cell depletion after anti-CD20 therapy. In B cell lymphomas, rituximab induces B cell depletion by three major mechanisms: antibodymediated cellular cytotoxicity (ADCC), whose importance is well-established, and complement-dependent cytotoxicity and antibody-triggered apoptosis, which are more controversial, both in animal models and in humans (30) (Figure 1). Thus, the multiple defects of the innate immune system in NOD mice may diminish the efficacy of anti-CD20 therapy. Indeed, NOD mice have defects in both inhibitory and activating Fc receptors (which are critical for ADCC) as well as complement pathways (31-33). Furthermore, NOD mice display defective monocyte/macrophages and NK cell function (34-36), which are important for phagocytosis and ADCC and may thus affect $B$ cell depletion following anti-CD20 treatment. In this regard, comparison of anti-CD20 therapy in mouse strains displaying distinct Fc receptor networks and ADCC capabilities may be useful in mimicking the polymorphism of $\mathrm{Fc}$ receptors in humans that can affect ADCC and tumor responses to rituximab (37). Hu et al. (1) make the interesting suggestion that, in hCD20/NOD mice, anti-CD20 treatment leads to the generation of Treg and regulatory B cell populations that can control diabetes in an adoptive transfer model (Figure 1). We and others have shown the crucial role of $\mathrm{CD} 4^{+} \mathrm{CD} 25^{+}$Tregs in the regulation of autoimmune diabetes in NOD mice (38-40). Thus, this cell population is likely a critical element in therapeutic settings in achieving long-term active regulation of disease. However, the increase in Foxp $3^{+}$Tregs reported by Hu et al. is quite modest, not clearly antigen specific, and it is still unclear whether this cell population offers any protection from diabetes following anti-CD20 therapy. Additionally, Hu et al. suggest that anti-CD20 therapy affects the distribution of B cell subsets after B cell repopulation and leads to an increase in 
transitional type 2 B cells that may have a regulatory function, reminiscent of IL-10producing "regulatory" B cells that could limit the severity of disease in autoimmunity (41). Although the current clinical data do not mention the occurrence of autoimmunity following rituximab treatment in lymphoma patients, the elimination of such regulatory $\mathrm{B}$ cells may be of concern in B cell-depleting therapies, as demonstrated by a recent case report describing the exacerbation of ulcerative colitis associated with decreased local IL-10 production in one patient following rituximab treatment (42). In contrast, the possibility that rituximab may favor the development of regulatory $\mathrm{B}$ cells in autoimmune diseases is quite interesting and warrants further investigation. Finally, in autoimmune diseases involving autoreactive $\mathrm{T}$ and $\mathrm{B}$ cells such as T1D, it may be worth considering combination immunotherapies that target both arms of the response, such as combining rituximab with $\mathrm{Fc}$ receptor-nonbinding anti-CD3 or Thymoglobulin (Genzyme Corp.) $(16,43)$. Indeed, Fc receptor-nonbinding anti-CD3 and Thymoglobulin (antithymocyte globulin) are T cell-targeting therapies believed to function primarily by altering $\mathrm{T}$ cell function. The effects of these drugs involve a combination of $T$ cell depletion and the generation of regulatory cell populations such as Tregs (44, $45)$. Both drugs have been shown to reverse diabetes in the NOD mouse model (16, 43). Importantly, Fc receptor-nonbinding anti-CD3 has generated encouraging data in experimental trials of T1D patients, and both drugs are currently in clinical trials in T1D $(46,47)$. Thus, a combination of rituximab and $\mathrm{T}$ cell-targeting therapies are being tested in animal models and humans, as they may offer a more comprehensive approach to T1D therapy by eliminating autoreactive $T$ and $B$ cells that cooperate to induce disease and generating both Treg and regulatory $B$ cell populations that may contribute to long-term remission and maintenance of tolerance $(1,44)$. However, safety is a key component in designing immunotherapies for T1D, since it often begins in young children, and one has to be careful not to trade off the imperfect management of diabetes and its complications with insulin for long-term and generalized immunosuppression.

\section{Acknowledgments}

The authors wish to thank Mark Pescovitz (Indiana School of Medicine) and Anthony
DeFranco (UCSF) for their critical reading of the manuscript.

Address correspondence to: Jeffrey A. Bluestone, UCSF Diabetes Center, HSW 1118, Box 0540, 513 Parnassus Avenue, San Francisco, California 94143-0540, USA. Phone: (415) 514-1683; Fax: (415) 564-5813; E-mail: jbluest@diabetes.ucsf.edu.

1. Hu, C., et al. 2007. Treatment with CD20-specific antibody prevents and reverses autoimmune diabetes in mice. J. Clin. Invest. 117:3857-3867.

2. Gorman, C., Leandro, M., and Isenberg, D. 2003. B cell depletion in autoimmune disease. Arthritis Res. Ther. 5(Suppl. 4):S17-S21.

3. Keogh, K.A., et al. 2006. Rituximab for refractory Wegener's granulomatosis: report of a prospective, open-label pilot trial. Am. J. Respir. Crit. Care Med. 173:180-187.

4. Leandro, M.J., Cambridge, G., Edwards, J.C., Ehrenstein, M.R., and Isenberg, D.A. 2005. B-cell depletion in the treatment of patients with systemic lupus erythematosus: a longitudinal analysis of 24 patients. Rheumatology (Oxford). 44:1542-1545.

5. Quartier, P., et al. 2001. Treatment of childhood autoimmune haemolytic anaemia with rituximab. Lancet. 358:1511-1513.

6. Stasi, R., Pagano, A., Stipa, E., and Amadori, S. 2001. Rituximab chimeric anti-CD20 monoclonal antibody treatment for adults with chronic idiopathic thrombocytopenic purpura. Blood. 98:952-957.

7. Tanaka, Y., et al. 2007. A multicenter phase I/II trial of rituximab for refractory systemic lupus erythematosus. Mod. Rheumatol. 17:191-197.

8. Cree, B. 2006. Emerging monoclonal antibody therapies for multiple sclerosis. Neurologist. 12:171-178.

9. Edwards, J.C., Cambridge, G., and Leandro, M.J. 2006. B cell depletion therapy in rheumatic disease. Best Pract. Res. Clin. Rheumatol. 20:915-928.

10. Anderson, M.S., and Bluestone, J.A. 2005. The NOD mouse: a model of immune dysregulation. Annu. Rev. Immunol. 23:447-485.

11. Makino, S., et al. 1980. Breeding of a non-obese, diabetic strain of mice. Jikken Dobutsu. 29:1-13.

12. Kikutani, H., and Makino, S. 1992. The murine autoimmune diabetes model: NOD and related strains. Adv. Immunol. 51:285-322.

13. Haskins, K., and McDuffie, M. 1990. Acceleration of diabetes in young NOD mice with a CD4+ isletspecific T cell clone. Science. 249:1433-1436.

14. Wong, F.S., Visintin, I., Wen, L., Flavell, R.A., and Janeway, C.A., Jr. 1996. CD8 T cell clones from young nonobese diabetic (NOD) islets can transfer rapid onset of diabetes in NOD mice in the absence of CD4 cells. J. Exp. Med. 183:67-76.

15. Shizuru, J.A., Taylor-Edwards, C., Banks, B.A., Gregory, A.K., and Fathman, C.G. 1988. Immunotherapy of the nonobese diabetic mouse: treatment with an antibody to T-helper lymphocytes. Science. 240:659-662.

16. Chatenoud, L., Thervet, E., Primo, J., and Bach, J.F. 1994. Anti-CD3 antibody induces long-term remission of overt autoimmunity in nonobese diabetic mice. Proc. Natl. Acad. Sci. U. S. A. 91:123-127.

17. Leslie, D., Lipsky, P., and Notkins, A.L. 2001. Autoantibodies as predictors of disease. J. Clin. Invest. 108:1417-1422.

18. Yu, L., et al. 2000. Early expression of antiinsulin autoantibodies of humans and the NOD mouse: evidence for early determination of subsequent diabetes. Proc. Natl. Acad. Sci. U. S. A. 97:1701-1706.

19. Greeley, S.A., et al. 2002. Elimination of maternally transmitted autoantibodies prevents diabetes in nonobese diabetic mice. Nat. Med. 8:399-402.

20. Serreze, D.V., et al. 1996. B lymphocytes are essen- tial for the initiation of T cell-mediated autoimmune diabetes: analysis of a new "speed congenic” stock of NOD.Ig mu null mice. J. Exp. Med. 184:2049-2053

21. Bour-Jordan, H., et al. 2007. Constitutive expression of B7-1 on B cells uncovers autoimmunity toward the B cell compartment in the nonobese diabetic mouse. J. Immunol. 179:1004-1012.

22. Falcone, M., Lee, J., Patstone, G., Yeung, B., and Sarvetnick, N. 1998. B lymphocytes are crucial antigen-presenting cells in the pathogenic autoimmune response to GAD65 antigen in nonobese diabetic mice. J. Immunol. 161:1163-1168.

23. Noorchashm, H., et al. 1999. I-Ag7-mediated antigen presentation by B lymphocytes is critical in overcoming a checkpoint in T cell tolerance to islet beta cells of nonobese diabetic mice. J. Immunol. 163:743-750.

24. Silveira, P.A., et al. 2002. The preferential ability of B lymphocytes to act as diabetogenic APC in NOD mice depends on expression of self-antigen-specific immunoglobulin receptors. Eur. J. Immunol. 32:3657-3666

25. NIAID and NIDDK. B-lymphocyte immunotherapy in islet transplantation. http://clinicaltrials. gov/ct/show/NCT00468442?order=1.

26. Hamaguchi, Y., et al. 2005. The peritoneal cavity provides a protective niche for $\mathrm{B} 1$ and conventional B lymphocytes during anti-CD20 immunotherapy in mice. J. Immunol. 174:4389-4399.

27. Gong, Q., et al. 2005. Importance of cellular microenvironment and circulatory dynamics in B cell immunotherapy. J. Immunol. 174:817-826.

28. NIDDK, NIAID, NICHD, American Diabetes Association, and Juvenile Diabetes Research Foundation. Effects of rituximab on the progression of type 1 diabetes in new onset subjects. http://clinicaltrials.gov/ct/show/NCT00279305?order $=1$.

29. Sherry, N.A., et al. 2007. Exendin-4 improves reversal of diabetes in NOD mice treated with anti-CD3 $\mathrm{mAb}$ by enhancing recovery of $\{$ beta $\}$ cells. Endocrinology. 148:5136-5144.

30. Glennie, M.J., French, R.R., Cragg, M.S., and Taylor, R.P. 2007. Mechanisms of killing by antiCD20 monoclonal antibodies. Mol. Immunol. 44:3823-3837.

31. Baxter, A.G., and Cooke, A. 1993. Complement lytic activity has no role in the pathogenesis of autoimmune diabetes in NOD mice. Diabetes. 42:1574-1578.

32. Gavin, A.L., Tan, P.S., and Hogarth, P.M. 1998. Gain-of-function mutations in FcgammaRI of NOD mice: implications for the evolution of the Ig superfamily. EMBO J. 17:3850-3857.

33. Luan, J.J., et al. 1996. Defective Fc gamma RII gene expression in macrophages of NOD mice: genetic linkage with up-regulation of $\operatorname{IgG} 1$ and $\operatorname{IgG} 2 \mathrm{~b}$ in serum. J. Immunol. 157:4707-4716.

34. Kataoka, S., et al. 1983. Immunologic aspects of the nonobese diabetic (NOD) mouse. Abnormalities of cellular immunity. Diabetes. 32:247-253.

35. Ogasawara, K., et al. 2003. Impairment of NK cell function by NKG2D modulation in NOD mice. Immunity. 18:41-51.

36. Serreze, D.V., Gaskins, H.R., and Leiter, E.H. 1993. Defects in the differenciation and function of antigen presenting cells in NOD/Lt mice. J. Immunol. 150:2534-2543.

37. Hatjiharissi, E., et al. 2007. Increased natural killer cell expression of CD16, augmented binding and ADCC activity to rituximab among individuals expressing the Fc\{gamma\}RIIIa-158 V/V and V/F polymorphism. Blood. 110:2561-2564

38. Bour-Jordan, H., et al. 2004. Costimulation controls diabetes by altering the balance of pathogenic and regulatory T cells. J. Clin. Invest. 114:979-987.

39. Salomon, B., et al. 2000. B7/CD28 costimulation is essential for the homeostasis of the CD4+CD25+ 
immunoregulatory $\mathrm{T}$ cells that control autoimmune diabetes. Immunity. 12:431-440.

40. Tang, Q., et al. 2006. Visualizing regulatory T cell control of autoimmune responses in nonobese diabetic mice. Nat. Immunol. 7:83-92.

41. Fillatreau, S., Sweenie, C.H., McGeachy, M.J., Gray, D., and Anderton, S.M. 2002. B cells regulate autoimmunity by provision of IL-10. Nat. Immunol. 3:944-950.

42. Goetz, M., et al. 2007. Exacerbation of ulcerative colitis after rituximab salvage therapy. Inflamm. Bowel Dis. 13:1365-1368.

43. Maki, T., Ichikawa, T., Blanco, R., and Porter, J. 1992. Long-term abrogation of autoimmune diabetes in nonobese diabetic mice by immunotherapy with anti-lymphocyte serum. Proc. Natl. Acad. Sci. U. S. A. 89:3434-3438.

44. Belghith, M., et al. 2003. TGF-beta-dependent mechanisms mediate restoration of self-tolerance induced by antibodies to CD3 in overt autoim- mune diabetes. Nat. Med. 9:1202-1208.

45. Mohty, M. 2007. Mechanisms of action of antithymocyte globulin: T-cell depletion and beyond. Leukemia. 21:1387-1394.

46. Herold, K.C., et al. 2002. Anti-CD3 monoclonal antibody in new-onset type 1 diabetes mellitus. N. Engl. J. Med. 346:1692-1698.

47. Immune Tolerance Network. Clinical trials for newly diagnosed type 1 diabetes. http://www. newonsetdiabetes.org/trials.html.

\title{
Every allograft needs a silver lining
}

\author{
Alan G. Contreras and David M. Briscoe
}

Transplantation Research Center and Division of Nephrology, Children's Hospital Boston, Boston, Massachusetts, USA. Department of Pediatrics, Harvard Medical School, Boston, Massachusetts, USA.

\begin{abstract}
The development of chronic allograft rejection is based on the hypothesis that cumulative, time-dependent tissue injury eventually leads to a fibrotic response. In this issue of the JCI, Babu and colleagues found that alloimmune-mediated microvascular loss precedes tissue damage in murine orthotopic tracheal allografts (see the related article beginning on page 3774 ). The concept that injury to the endothelium may precede airway fibrosis suggests that interventions to maintain vascular integrity may be important, especially in the case of lung transplantation. Further, for all solid organ allografts, it is possible that the key to long-term allograft survival is physiological vascular repair at early times following transplantation.
\end{abstract}

\section{Clues to mechanisms underlying long-term allograft survival}

The major obstacle to the long-term survival of lung transplant recipients is the development of the bronchiolitis obliterans syndrome (BOS), which typically occurs in up to $60 \%$ of patients who survive five years (1). BOS is considered a major posttransplant complication and is characterized by a progressive luminal airway narrowing and histological evidence of fibrosis. Pathological features of BOS suggest that injury and inflammation of epithelial cells and subepithelial structures within small airways lead to the fibrosis response. Moreover, it is thought that repetitive injury in lung allografts, as in other solid organ transplants (e.g., kidney) $(2,3)$, may elicit cumulative, time-dependent damage and result in cellular atrophy and chronic interstitial fibrosis (1). According to this paradigm, prevention or inhibition of injury (using time-dependent therapies) should result in long-term graft survival (4).

Nonstandard abbreviations used: BOS, bronchiolitis obliterans syndrome.

Conflict of interest: David M. Briscoe has received research funding and/or has served as a consultant to the Roche Organ Transplantation Research Foundation and Wyeth Pharmaceuticals.

Citation for this article: J. Clin. Invest. 117:3645-3648 (2007). doi:10.1172/JCI34238.
Nevertheless, for all solid organ allografts, an underappreciated aspect of the injury process is its association with repair. It has been proposed that augmenting physiological repair, even in the face of injury, will ensure that the end result of injury is limited $(5,6)$. On the other hand, if the repair process is inhibited, then even milder forms of injury have the potential to result in extensive tissue damage. Therefore, it is possible that an understanding of repair is key to long-term allograft survival.

In this issue of the JCI, Babu et al. set out to address whether the maintenance of microvasculature integrity is sufficient to sustain lung allograft function and to prevent the development of fibrosis/chronic rejection (7). These authors used a murine orthotopic tracheal transplantation model and found that alloimmune-mediated microvascular loss results in hypoxia, which precedes tissue damage and the development of intragraft fibrosis in the recipient trachea. If endothelial cell injury was inhibited (by limiting ongoing inflammation) and the microvasculature was maintained intact, physiological remodeling occurred and allograft tissue morphology in these animals returned to normal (Figure 1). Therefore, in general, once an allograft is revascularized and is functioning, protect- ing the vasculature and/or enabling physiological homeostatic repair of the microvasculature will prevent tissue fibrosis.

\section{Role of microvascular repair in the preservation of allografts}

In the context of transplantation, it should be emphasized that microvascular endothelial cells are very susceptible to injury, including changes in oxygen tension (hypoxic injury), reperfusion injury, and oxidative stress, as well as persistent episodes of silent rejection (8). These repetitive insults clearly target the microvasculature and create a circumstance whereby endothelial loss and/or damage will typically occur $(9,10)$. Within allografts, the loss of the microvasculature results in impaired delivery of oxygen and nutrients to key organ-specific cells, such as columnar epithelial cells in the lung or tubular epithelial cells in the kidney, which in turn results in chronic ischemia and cell death $(5,6,8,11)$. If vascular integrity is coincidently compromised, recovery may not be complete and/or may not occur, and cellular atrophy will lead to progressive functional tissue loss. The implication of these observations is that the limiting factor in the allograft injury process is inefficient microvascular repair. This concept may be particularly relevant following lung transplantation, where bronchial artery vascular reanastomosis is often not done, and thus vascular integrity may be easily compromised at early times as a result of alloimmune attack. Indeed, microvascular injury has been documented in association with rejection (12), and replacement of endothelial cells correlates with degrees of injury in human allografts $(8,13,14)$. Therefore, one must assume that the health 